Research article

\title{
Systematic reviews of complementary therapies - an annotated bibliography. Part 2: Herbal medicine Klaus Linde ${ }^{* 1,2}$, Gerben ter Riet ${ }^{3,4}$, Maria Hondras ${ }^{5}$, Andrew Vickers ${ }^{6}$, Reinhard Saller ${ }^{7}$ and Dieter Melchart ${ }^{1}$
}

Address: ${ }^{1}$ Centre for Complementary Medicine Research, Department of Internal Medicine II, Technische Universität, München, Kaiserstr. 9, 80801 München, Germany, ${ }^{2}$ Institute for Social Medicine \& Epidemiology, Charité Hospital, Humboldt University, Berlin, Germany, ${ }^{3} \mathrm{HS}$ Centre for Reviews \& Dissemination, University of York, UK, ${ }^{4}$ Department of Epidemiology, Maastricht University, The Netherlands, ${ }^{5}$ Consortial Center for Chiropractic Research, Davenport, Iowa, USA, ${ }^{6}$ Memorial Sloan-Kettering Cancer Center, New York, USA and ${ }^{7}$ Division of Complementary Medicine Department of Internal Medicine, Universitätsspital Zurich, Switzerland

E-mail: Klaus Linde* - Klaus.Linde@lrz.tu-muenchen.de; Gerben ter Riet - GterRiet@EPID.UNIMAAS.NL;

Maria Hondras - mhondras@interaccess.com; Andrew Vickers - vickersa@mskcc.org; Reinhard Sailer - reinhard.saller@dim.usz.ch; Dieter Melchart - Dieter.Melchart@lrz.tu-muenchen.de

${ }^{*}$ Corresponding author

Published: 20 July 2001

BMC Complementary and Alternative Medicine 200I, I:5
Received: 22 March 200I

Accepted: 20 July 2001

This article is available from: http://www.biomedcentral.com/1472-6882/1/5

(C) 200I Linde et al; licensee BioMed Central Ltd. Verbatim copying and redistribution of this article are permitted in any medium for any non-commercial purpose, provided this notice is preserved along with the article's original URL. For commercial use, contact info@biomedcentral.com

\begin{abstract}
Background: Complementary therapies are widespread but controversial. We aim to provide a comprehensive collection and a summary of systematic reviews of clinical trials in three major complementary therapies (acupuncture, herbal medicine, homeopathy). This article is dealing with herbal medicine. Potentially relevant reviews were searched through the register of the Cochrane Complementary Medicine Field, the Cochrane Library, Medline, and bibliographies of articles and books. To be included articles had to review prospective clinical trials of herbal medicines; had to describe review methods explicitly; had to be published; and had to focus on treatment effects. Information on conditions, interventions, methods, results and conclusions was extracted using a pre-tested form and summarized descriptively.
\end{abstract}

Results: From a total of 79 potentially relevant reviews pre-selected in the screening process 58 met the inclusion criteria. Thirty of the reports reviewed ginkgo (for dementia, intermittent claudication, tinnitus, and macular degeneration), hypericum (for depression) or garlic preparations (for cardiovascular risk factors and lower limb atherosclerosis). The quality of primary studies was criticized in the majority of the reviews. Most reviews judged the available evidence as promising but definitive conclusions were rarely possible.

Conclusions: Systematic reviews are available on a broad range of herbal preparations prescribed for defined conditions. There is very little evidence on the effectiveness of herbalism as practised by specialist herbalists who combine herbs and use unconventional diagnosis.

\section{Introduction}

In this second part of our series on systematic reviews in complementary therapies we report our findings on herbal medicines. Herbal medicines (defined as preparations derived from plants and fungi, for example by alcoholic extraction or decoction, used to prevent and treat 
diseases) are an essential part of traditional medicine in almost any culture [1]. In industrialized countries herbal drugs and supplements are an important market. Some countries like Germany have a long tradition in the use of herbal preparations marketed as drugs and figures for prescriptions and sales are stable or slightly declining [2]. In the US and the UK herbal medicinal products are marketed as "food supplements" or "botanical medicines". In recent years sales of such products have been increasing strongly in these countries [3,4]. In the Third World herbs are mainly used by traditional healers [5].

\section{Methods}

A detailed description of the methods used in this review of reviews is given in the first part of this series [6]. For searches in Medline 50 single plant names and the 'exploded' term 'medicinal plants' were combined with the standard search strategy for systematic reviews. As a specific intervention-related inclusion criterion we required that reports reviewed prospective (not necessarily controlled) clinical trials of substances extracted from plants in humans. Reviews dealing with single substances (e.g., artemisin derivatives) derived from plants were excluded on the grounds that such agents are comparable to conventional drugs. Disease-oriented reviews including a variety of interventions were included only if they reviewed at least 4 herbal medicine trials.

\section{Results}

From a total of 79 potentially relevant reviews preselected in the literature screening process, 58 (published in 65 papers) met the inclusion criteria [7-71]. Eleven reports were not truly systematic reviews (not meeting inclusion criterion 2) [72-82], 5 dealt with isolated substances of plant origin [83-87] and 4 were excluded for other reasons (one disease- focused review with less than 4 herbal medicine trials [88], one review not on preventative or therapeutic use [89], two reviews not truly herbal medicine $[90,91])$.

More than half of the reports reviewed gingko, hypericum or garlic preparations. No less than 13 systematic reviews dealed with ginkgo (Ginkgo biloba) extracts (see table 1). Seven of these reviewed trials (total number of trials covered in any of the reviews 15) in patients with intermittent claudication [7-13]. Most of these reviews concluded that ginkgo extracts were significantly more effective than placebo in increasing measures like walking distance but the clinical relevance of the effects was felt to be moderate by some reviewers. The five reviews dealing with dementia and cerebral insufficiency (total number of trials included about 50) all draw positive conclusions [13-17]. However, many of the older trials were in patients with minor cognitive impairment and more evidence is needed to decide whether ginkgo ex- tracts have clinically relevant beneficial effects in more severe forms of dementia. Finally, one review found that ginkgo extracts might be effective in the treatment of tinnitus [18] and another found insufficient evidence for efficacy in patients with macular degeneration [19].

The effectiveness of St. John's wort (Hypericum perforatum) extracts in depression was investigated in nine reviews [20-30] (total number of trials covered 29; see table 2). Mainly due to slight differences in the inclusion criteria (for example, restriction to trials with a minimum of 6 weeks observation or with a minimum quality score) the respective study collections differed to a considerable amount. However, the conclusions were very similar. Hypericum extracts have been shown to be superior to placebo in mild to moderate depressive disorders. There is growing evidence that hypericum is as effective as other antidepressants for mild to moderate depression and causes fewer side effects but further trials are still needed to establish long-term effectiveness and safety.

Eight reviews have been performed on garlic (Allium sativum) for cardiovascular risk factors [31-38] (total number of trials covered about 50) and lower limb atherosclerosis [39] (see table 2). A modest short-term effect over placebo on lipid-lowering seems to be established but the clinical relevance of these effects is uncertain. Data from randomised trials on cardiovascular mortality are not available. Effects on blood pressure seem to be at best minor. The available results on fibrinolytic activity and platelet aggregation are promising but insufficient to draw clear conclusions. A specific problem in research on garlic is the great variety of garlic preparations used: the exact content of bioactive ingredients in these is often unclear.

Three reviews (covering a total of about 30 trials) have been performed on preparations containing extracts of Echinacea (Echinacea purpurea, pallida or angustifolia), two of which by the same study group [40-43]. The results suggest that Echinacea preparations may have some beneficial effects mainly in the early treatment of common colds. Similar to garlic a major problem is the high variaton of bioactive compounds between different Echinacea preparations. Cranberries (Vaccinium macrocarpon) for urinary tract infections [44,45], mistletoe (Viscum album) for cancer [46-48], peppermint (Mentha piperita) oil for irritable bowel syndromes [49,50] and saw palmetto (Serenoa repens) for benign prostate hyperplasia [51-53] have each been subject to two reviews. For saw palmetto there is good evidence for efficacy over placebo while for the other three the data are inconclusive (see table 3). 
Table I: Systematic reviews of clinical trials of ginkgo biloba extracts

\begin{tabular}{llcc}
\hline & & Features \\
Author Year Indication Intervention Comparisons Studies & $1 / 2 / 3 /$ & Results \\
$4 / 5$ & Author's Conclusion
\end{tabular}

\section{Ginkgo (Ginkgo biloba)}

Pittler 2000 intermittent

[7] claudication

placebo

8 RCT

$[8]$

Ernst 96 [9] intermittent

claudication

$\begin{array}{lll}\text { ginkgo } & \text { placebo, } & 10 \\ \text { extract } & \text { other drugs } & \text { RCT/CCT } \\ \text { EGb76I } & & \end{array}$

Schneider 92 intermittent

[10] claudication

ginkgo

placebo,

other

treatment

y/y/

Increase of pain-free walking

y/y

distance over placebo after

I 2 or 24 weeks $34 \mathrm{~m}(95 \% \mathrm{Cl}$

26-43 m)

y/y/yl Increase of pain-free walking

$\mathrm{n} / \mathrm{y} \quad$ distance over placebo after 24

weeks $32 \mathrm{~m}(95 \% \mathrm{Cl} \mathrm{I4-50} \mathrm{m)}$

$\mathrm{p} / \mathrm{p} / \mathrm{n} /$

$\mathrm{n} / \mathrm{n}$

Most studies low quality. In-

crease of walking distance compared to placebo 24 to 160

$\mathrm{m}$. At least similar effectiveness compared to other drugs.
Letzel 92 intermitent

[II] claudication tract EGb

761

Kleijnen 9I intermittent ginkgo

[12] claudication

plac., ginkgo

pentoxifyllin $9 \mathrm{RCT}$

vs. plac. pentoxifyllin

ginkgo vs.

plac.,

pentoxifyllin (ginkgo), 5

vs. placebo

$\begin{array}{lll}\text { Weiss 91 } & \begin{array}{l}\text { cerebral } \\ \text { ins., }\end{array} & \text { ginkgo } \\ & \begin{array}{l}\text { intermittent } \\ \text { claudication }\end{array} & \text { Extract } \\ \text { [13] } & \text { EG76I }\end{array}$

Ernst 99 [14] dementia gink

$\begin{array}{lllll}\begin{array}{l}\text { Oken } 98 \\ \text { [15] }\end{array} & \begin{array}{l}\text { Alzheimer } \\ \text { dementia }\end{array} & \text { ginkgo } & \text { placebo } & 4 \text { RCT }\end{array}$

$\begin{array}{lll}\begin{array}{l}\text { Hopfen- } \\ \text { müller } \\ 94[16]\end{array} & \text { cerebral } & \text { ginkgo } \\ & \text { insufficiency } & \begin{array}{l}\text { extract LI } \\ 1370\end{array} \\ \begin{array}{lll}\text { Kleijnen } 92 & \begin{array}{l}\text { cerebral } \\ \text { [17] }\end{array} & \text { ginkgufficiency }\end{array} \\ & & \end{array}$

placebo I7RCT/

CCT

(cerebral

ins.), 8

$\mathrm{RCT} / \mathrm{CCT}$

placebo

9 RCT

RCT

placebo

I0 RCT, I

CCT

ginkgo vs.

plac.
$7 \mathrm{RCT} / \mathrm{CCT}$

$? / \mathrm{n} / \mathrm{n} /$

(vs. plac.), $2 \quad y / y$

15

$\mathrm{RCT} / \mathrm{CCT}$

pentoxif.

mean effect size $d=0.75$

$(95 \% \mathrm{Cl} 0.44-1.07)$ over placebo

?/p/n/ Pooled increase of walking

y/y distance: $45 \%$ over placebo for gingko and $57 \%$ for pentoxifyllin

y/y/y/ Many trials low quality. All trials

$\mathrm{n} / \mathrm{n} \quad$ with positive results. Evidence

similar as for pentoxifyllin

?/p/p/ $\quad 10$ of 12 interpretable trials on

$\mathrm{n} / \mathrm{n}$

cerebral insufficieny and all 4

interpretable trials on

intermittent claudication with

significant positive results

$y / y / y / \quad$ Results collectively suggest

$\mathrm{y} / \mathrm{n} \quad$ that ginkgo is more effective for dementia than placebo

$y / y / n / \quad$ Significant effect over placebo

y/y for cognitive function (Hedges

$g=0.4 \mathrm{I}, 95 \% \mathrm{Cl} 0.22-0.6 \mathrm{I})$

$\mathrm{n} / \mathrm{n} / \mathrm{n} /$ Global response (based on

y/y

symptom scores): OR 1.98

(95\%CII.39-2.57) in favour of

Ginkgo

$40 \mathrm{RCT} / \quad \mathrm{y} / \mathrm{y} / \mathrm{y} /$

Many trials low quality. Virtually all trials reported positive
Evidence for a modest benefit of uncertain clinical relevance

Inconsistent results from the few available small studies do not allow firm conclusions

Available evidence promising but further high quality research needed.

Effectiveness over placebo clearly shown

Ginkgo extract EGb76I more effective than placebo and similarly effective as pentoxifyllin

Ginkgo seems effective for intermittent claudication but further high quality studies are needed

Effectiveness for both conditions

biometrically shown

Encouraging findings warranting

large scale trials

Clinical relevance of the observed

effects has to be confirmed in

further research

Ginkgo extract superior to placebo

Ginkgo seems effective for cerebral insufficiency but further 
Table I: Systematic reviews of clinical trials of ginkgo biloba extracts (Continued)

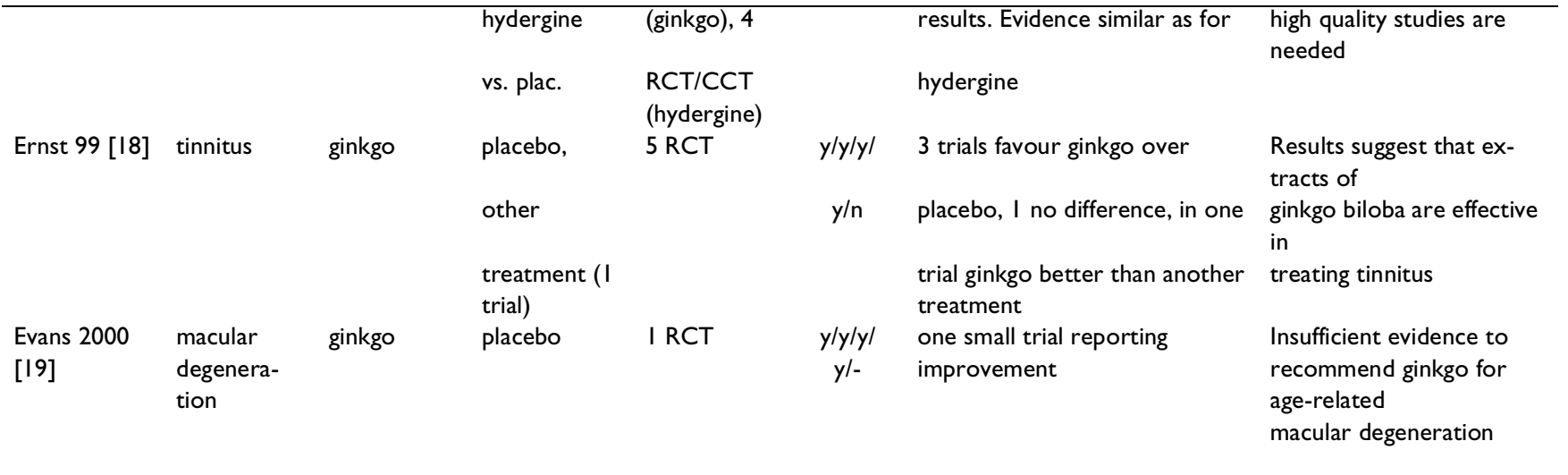

Features: I = comprehensive search, 2 = explicit inclusion criteria, 3 = formal quality assessment, $4=$ summary of results for each included study, 5 = meta-analysis; $y=$ yes, $\mathrm{p}=$ partly, $\mathrm{n}=$ no, - = not applicable, ? = unclear review on all pharmacologic treatments for the respective condition $\mathrm{RCT}$ $=$ randomized controlled trials, $\mathrm{CCT}=$ non-randomized controlled trials, $\mathrm{CS}=$ cohort studies, UCS $=$ uncontrolled studies; $\mathrm{OR}=$ odds ratio, $\mathrm{RR}=$ rate ratio

Single systematic reviews have been published on aloe (Aloe vera) [54], artichoke (Cynara scolymus) leave extract [55], evening primrose (Oenothera biennis) oil [56], feverfew (Tanacetum parthenium) [57], ginger (Zingiber officinialis) [58], ginseng (Panax ginseng) [59], horse chestnut (Aesculus hippocastanum) seeds [60], kava (Piper methysticum) [61], milk thistle (Silybum marianum) [62], a fixed combination of three herbal extracts [63], rye-grass pollen (Secale cereale) extract $[64,65]$, tea tree (Melaleuca alternafolia) oil [66], and valerian (Valehana officinalis) root [67] (see table 4).
The only review which focused on a herbal intervention which is not marketed as a drug or food supplement was on cabbage leaves for breast engorgement and included a single small-scale trial [68]. Chinese herbal therapy for atopic eczema [69] and a variety of herbs for lowering blood glucose [70] and for analgesic and anti-inflammatory purposes [71] have also been reviewed. For some of these herbal preparations the evidence is promising but further studies are considered necessary to establish efficacy in almost every case.

Table 2: Systematic reviews of clinical trials of hypericum and garlic preparations

\begin{tabular}{|c|c|c|c|c|c|c|c|}
\hline \multicolumn{8}{|c|}{ Features } \\
\hline $\begin{array}{l}\text { Author } \\
\text { Year }\end{array}$ & Indication & Intervention & Comparisons & Studies & $\begin{array}{c}1 / 2 / 3 / \\
4 / 5\end{array}$ & Results & Author's Conclusion \\
\hline \multicolumn{8}{|c|}{ St John's wort (Hypericum perforatum) } \\
\hline \multirow[t]{2}{*}{$\begin{array}{l}\text { Gaster } \\
2000[20]\end{array}$} & depression & hypericum & $\begin{array}{l}\text { placebo and } \\
\text { antidepres- } \\
\text { sants }\end{array}$ & $8 \mathrm{RCT}$ & $\begin{array}{l}\mathrm{p} / \mathrm{y} / \mathrm{p} / \\
\mathrm{y} / \mathrm{n}\end{array}$ & $\begin{array}{l}4 \text { placebo-controlled trials with } \\
\text { positive results, in } 4 \text { trials }\end{array}$ & $\begin{array}{l}\text { Data suggest that hypericum is } \\
\text { superior to placebo, insuf- } \\
\text { fcient }\end{array}$ \\
\hline & & & & & & $\begin{array}{l}\text { standard antidepr. tended to be } \\
\text { slightly better }\end{array}$ & $\begin{array}{l}\text { evidence re equivalence with } \\
\text { antidepressants }\end{array}$ \\
\hline $\begin{array}{l}\text { Williams } \\
2000 \&\end{array}$ & depression & $\begin{array}{l}\text { hypericum } \\
\text { (and other }\end{array}$ & $\begin{array}{l}\text { placebo and } \\
\text { antidepres- } \\
\text { sants }\end{array}$ & I4 RCT & $\begin{array}{l}y / y / n / \\
y / y\end{array}$ & $\begin{array}{l}\text { Treatment response: RR I.9 } \\
(95 \% \mathrm{CI} I .2-2.8) \text { vs. placebo and }\end{array}$ & $\begin{array}{l}\text { Data suggest that hypericum is } \\
\text { superior to placebo, insuf- } \\
\text { fcient }\end{array}$ \\
\hline $\begin{array}{l}\text { Mulrow } 98 \\
{[21,22]}\end{array}$ & & drugs) & & & & I.2(I.0-I.4) vs. antidepressants & $\begin{array}{l}\text { evidence re equivalence with } \\
\text { antidepressants }\end{array}$ \\
\hline \multirow[t]{3}{*}{ Kim 99 [23] } & depression & hypericum & placebo and & $6 \mathrm{RCT}$ & $\mathrm{p} / \mathrm{y} / \mathrm{y} /$ & Treatment response: RR I.48 & $\begin{array}{l}\text { Hypericum more effective } \\
\text { than }\end{array}$ \\
\hline & & & $\begin{array}{l}\text { antidepres- } \\
\text { sants }\end{array}$ & & $y / y$ & (95\%Cl I.03-1.92) vs. placebo & $\begin{array}{l}\text { placebo and similarly effective } \\
\text { as }\end{array}$ \\
\hline & & & & & & $\begin{array}{l}\text { and } 0.98(0.67-1.28) \text { vs. } \\
\text { antidepressants }\end{array}$ & $\begin{array}{l}\text { low dose antidepressants; } \\
\text { quality problems }\end{array}$ \\
\hline
\end{tabular}


Table 2: Systematic reviews of clinical trials of hypericum and garlic preparations (Continued)

\begin{tabular}{|c|c|c|c|c|c|c|c|}
\hline Stevinson & depression & hypericum & placebo and & $6 \mathrm{RCT}$ & $y / y / y /$ & Only trials published after Linde & $\begin{array}{l}\text { Data confirm findings of earli- } \\
\text { er }\end{array}$ \\
\hline \multirow[t]{2}{*}{$99[24]$} & & & $\begin{array}{l}\text { antidepres- } \\
\text { sants }\end{array}$ & & $y / n$ & 96; trials show effects better & $\begin{array}{l}\text { trials, but still insuff. evidence } \\
\text { to }\end{array}$ \\
\hline & & & & & & $\begin{array}{l}\text { than placebo/similar to } \\
\text { antidepressants }\end{array}$ & $\begin{array}{l}\text { assess equivalence with } \\
\text { antidepressants }\end{array}$ \\
\hline Linde 98 \& & depression & hypericum & placebo and & $27 \mathrm{RCT}$ & $y / y / y /$ & Treatment response: RR 2.47 & $\begin{array}{l}\text { Hypericum more effective } \\
\text { than }\end{array}$ \\
\hline \multirow[t]{2}{*}{$96[25,26]$} & & & $\begin{array}{l}\text { antidepres- } \\
\text { sants }\end{array}$ & & y/y & $(95 \% C I I .69-3.6 I)$ vs. placebo & $\begin{array}{l}\text { placebo. Inadequate evidence } \\
\text { to }\end{array}$ \\
\hline & & & & & & $\begin{array}{l}\text { and I.0I (0.87-I.I6) vs. } \\
\text { antidepressants }\end{array}$ & $\begin{array}{l}\text { assess equivalence with } \\
\text { antidepressants }\end{array}$ \\
\hline Volz 97 & depression & hypericum & placebo and & 15 & $\mathrm{p} / \mathrm{p} / \mathrm{n} /$ & Most placebo-controlled trials & $\begin{array}{l}\text { A therapy with hypericum of } \\
\text { mild }\end{array}$ \\
\hline$[27]$ & & & $\begin{array}{l}\text { antidepres- } \\
\text { sants }\end{array}$ & $\begin{array}{l}\mathrm{RCT} / \\
\mathrm{CCT}\end{array}$ & $\mathrm{n} / \mathrm{n}$ & $\begin{array}{l}\text { positive; similarly effective as } \\
\text { (not adequately dosed) antide- } \\
\text { pressants }\end{array}$ & $\begin{array}{l}\text { and moderate depression can } \\
\text { be attempted. Further studies } \\
\text { needed }\end{array}$ \\
\hline Ernst 95 & depression & hypericum & placebo and & II RCT & y/y/y/ & Most of 8 placebo-controlled & $\begin{array}{l}\text { Hypericum is superior to pla- } \\
\text { cebo }\end{array}$ \\
\hline [28] & & & $\begin{array}{l}\text { antidepres- } \\
\text { sants }\end{array}$ & & $y / n$ & $\begin{array}{l}\text { trials positive. } 3 \text { trials against } \\
\text { standard medication with similar } \\
\text { effects }\end{array}$ & $\begin{array}{l}\text { and seems equally effective as } \\
\text { standard medication }\end{array}$ \\
\hline $\begin{array}{l}\text { Volz } 2000 \\
\text { [29] }\end{array}$ & $\begin{array}{l}\text { mild to } \\
\text { mod. } \\
\text { depression }\end{array}$ & hypericum & fluoxetine & $\begin{array}{l}\mathrm{I} 7+9 \\
\mathrm{CCT}\end{array}$ & $\begin{array}{c}\mathrm{n} / \mathrm{y} / \mathrm{n} / \\
\mathrm{y} / \mathrm{n}\end{array}$ & $\begin{array}{l}\text { No direct comparison of } \\
\text { hypericum and fluoxetine } \\
\text { available. Mean depression score } \\
\text { (HAMD) reduction in hypericum } \\
\text { trials } 53 \% \text {, in fluoxetine trials } \\
55 \%\end{array}$ & $\begin{array}{l}\text { Response rates are similar; } \\
\text { findings difficult to interpret } \\
\text { because of the indirect com- } \\
\text { parison }\end{array}$ \\
\hline Friede 98 & anxiety in & hypericum & placebo, & $8 \mathrm{RCT}$ & $? / y / y /$ & $\begin{array}{l}\text { Trials collectively show reduc- } \\
\text { tion }\end{array}$ & Hypericum is effective for \\
\hline [30] & $\begin{array}{l}\text { depressed } \\
\text { p. }\end{array}$ & & amitriptyline & & $y / n$ & $\begin{array}{l}\text { of anxiety symptoms over place- } \\
\text { bo. Only I trial vs amitriptyline }\end{array}$ & $\begin{array}{l}\text { depressed patients with anxie- } \\
\text { ty }\end{array}$ \\
\hline \multicolumn{8}{|c|}{ Garlic (Allium sativum) } \\
\hline Lawrence & cardiovasc. & garlic & $\begin{array}{l}\text { mainly place- } \\
\text { bo; }\end{array}$ & $45 \mathrm{RCT}$ & y/y/y/ & 37 trials consistently show small & $\begin{array}{l}\text { Insufficient data to draw con- } \\
\text { clusion }\end{array}$ \\
\hline $2000[31]$ & risk factors & & $\begin{array}{l}\text { no \& other } \\
\text { treatment }\end{array}$ & & $y / y$ & $\begin{array}{l}\text { short-term effects over placebo } \\
\text { for cholesterol reduction. No } \\
\text { consistent effects on blood pres- } \\
\text { sure, promising effects re } \\
\text { platelet aggregation and fibriono- } \\
\text { lytic activity }\end{array}$ & $\begin{array}{l}\text { regarding clinical cardiovascu- } \\
\text { lar outcomes. Garlic prepara- } \\
\text { tions may have small, positive, } \\
\text { short-term effects on lipids }\end{array}$ \\
\hline Stevinson & hyperchol- & garlic & placebo & I3 RCT & y/y/y/ & Pooled total cholesterol & $\begin{array}{l}\text { Available data suggest that gar- } \\
\text { lic is }\end{array}$ \\
\hline \multirow[t]{3}{*}{2000 [32] } & $\begin{array}{l}\text { esterolem- } \\
\text { ia }\end{array}$ & & & & y/y & reduction over placebo $0.4 \mathrm{I}$ & $\begin{array}{l}\text { superior to placebo. The size } \\
\text { of the }\end{array}$ \\
\hline & & & & & & $(95 \% \mathrm{Cl}-0.66$ to -0.15$) \mathrm{mmol} / \mathrm{l}$ & $\begin{array}{l}\text { effect is modest. The use of } \\
\text { garlic }\end{array}$ \\
\hline & & & & & & $\begin{array}{l}\text { when analysis restricted to high } \\
\text { quality trials } 0.11 \text { ( }-0.30 \text { to } 0.08)\end{array}$ & $\begin{array}{l}\text { for hyperchol. is therefore of } \\
\text { questionable value }\end{array}$ \\
\hline $\begin{array}{l}\text { Silagy } 94 \& \\
\text { Neil } 96\end{array}$ & $\begin{array}{l}\text { cholesterol } \\
\text { lowering }\end{array}$ & garlic & placebo & I6 RCT & $\begin{array}{l}y / p / y / \\
y / y\end{array}$ & $\begin{array}{l}\text { Pooled cholesterol reduction } \\
\text { over placebo } 0.65(95 \% \mathrm{Cl} 0.53-\end{array}$ & $\begin{array}{l}\text { Meta-analysis suggests positive } \\
\text { effects but reviewers are scep- } \\
\text { tic }\end{array}$ \\
\hline$[33,34]$ & & & & & & $0.76) \mathrm{mmol} / \mathrm{l}$ & $\begin{array}{l}\text { (low quality; own replication } \\
\text { negative) }\end{array}$ \\
\hline Warshafsky & cholesterol & garlic & placebo & $5 \mathrm{RCT}$ & $\mathrm{p} / \mathrm{y} / \mathrm{y} /$ & Pooled cholesterol reduction & $\begin{array}{l}\text { Available evidence supports } \\
\text { the }\end{array}$ \\
\hline $93[35]$ & lowering & & & & y/y & $\begin{array}{l}\text { over placebo } 0.59(95 \% \mathrm{Cl} 0.44- \\
0.74) \mathrm{mmol} / \mathrm{l}\end{array}$ & $\begin{array}{l}\text { use of garlic as one modality to } \\
\text { decrease cholesterol levels }\end{array}$ \\
\hline Silagy 94 & lowering & dried garlic & placebo, other & $8 \mathrm{RCT}$ & y/p/y/ & Pooled reduction over placebo: & $\begin{array}{l}\text { Garlic maybe of some clinical } \\
\text { use }\end{array}$ \\
\hline
\end{tabular}


Table 2: Systematic reviews of clinical trials of hypericum and garlic preparations (Continued)

\begin{tabular}{|c|c|c|c|c|c|c|c|}
\hline [36] & blood & (Kwai) & treatment & & $y / y$ & SBP 7.7 (95\% Cl 4.3-II.0), DBP & $\begin{array}{l}\text { in subjects with mild hyperten- } \\
\text { sion. }\end{array}$ \\
\hline & press. & & & & & $5.0(2.9-7.1) \mathrm{mm} \mathrm{Hg}$ & Further research needed \\
\hline Kleijnen 9I & cardiovasc. & garlic & placebo & 18 & $\mathrm{p} / \mathrm{p} / \mathrm{y} /$ & Most studies with shortcomings. & No clear conclusion drawn \\
\hline$[37]$ & risk factors & supplements & & $\begin{array}{l}\mathrm{RCT} / \\
\mathrm{CCT}\end{array}$ & $y / n$ & $\begin{array}{l}\text { The majority of trials with pos. } \\
\text { results but inconsistent effect siz- } \\
\text { es }\end{array}$ & \\
\hline Kleijnen 89 & cardiovasc. & garlic \& & unclear & $\begin{array}{l}10 \\
\mathrm{RCT}\end{array}$ & $\mathrm{y} / \mathrm{p} / \mathrm{n} /$ & All trials with severe & Inadequate evidence to justify \\
\hline [38] & risk factors & onions & & $8 \mathrm{CCT}$ & $y / n$ & shortcomings. Fresh garlic with & $\begin{array}{l}\text { supplementation, further re- } \\
\text { search }\end{array}$ \\
\hline & & & & & & $\begin{array}{l}\text { beneficial effcts, onions and } \\
\text { commercially available } \\
\text { supplements yielded } \\
\text { contradictory results }\end{array}$ & needed \\
\hline $\begin{array}{l}\text { Jepson } 97 \\
\text { [39] }\end{array}$ & $\begin{array}{l}\text { lower limb } \\
\text { athero- } \\
\text { scler. }\end{array}$ & garlic & placebo & I RCT & $\begin{array}{c}y / y / y / \\
y /-\end{array}$ & $\begin{array}{l}\text { Walking distance not } \\
\text { significantly different between } \\
\text { groups }\end{array}$ & Insufficient evidence \\
\hline
\end{tabular}

legend see table I

Table 3: Systematic reviews of clinical trials of herbal medicines (at least 2 reviews per herb)

\begin{tabular}{llcc} 
Author Indication Intervention Comparisons Studies & $\begin{array}{c}\text { Features } \\
\mathrm{I} / 2 / 3 / \\
4 / 5\end{array}$ \\
Year & & Results Author's Conclusion & \\
\hline
\end{tabular}

\begin{tabular}{|c|c|c|c|c|c|c|c|}
\hline \multicolumn{8}{|c|}{ Echinacea (Echinacea purpurea, angustifolia and pallida) } \\
\hline Barrett & $\begin{array}{l}\text { upper re- } \\
\text { sp. }\end{array}$ & echinacea & placebo & $\begin{array}{l}\text { I } 3 R C \\
T\end{array}$ & $y / p / y /$ & Overall quality modest. All 4 & Echinacea may be beneficial for \\
\hline $99[40]$ & infections & $\begin{array}{l}\text { (incl. combi- } \\
\text { nations) }\end{array}$ & & & $y / n$ & $\begin{array}{l}\text { prevention studies show only } \\
\text { minor trends, } 8 \text { of } 9 \text { treatment } \\
\text { studies with generally positive } \\
\text { results }\end{array}$ & $\begin{array}{l}\text { early treatment of acute upper respi- } \\
\text { ratory infections; little evidence to } \\
\text { support the prolonged use for pre- } \\
\text { vention }\end{array}$ \\
\hline Melchart & common & echinacea & placebo, no & $\begin{array}{l}16 \\
\mathrm{RCT}\end{array}$ & $y / y / y /$ & Minor effects in prevention and & Echinacea extract can be efficacious \\
\hline $99[4 I]$ & cold & $\begin{array}{l}\text { (incl. } \\
\text { combina- } \\
\text { tions) }\end{array}$ & treatment & & $y / p$ & $\begin{array}{l}\text { treatment, promising effects in } \\
\text { early treatment. Heterogen. } \\
\text { preparations }\end{array}$ & $\begin{array}{l}\text { for the common cold, but evidence } \\
\text { insufficient for recommendations }\end{array}$ \\
\hline Melchart & immuno- & echinacea & placebo, no & $\begin{array}{l}18 \\
\text { RCT, } 8\end{array}$ & $y / y / y /$ & Most studies low quality. Most & Echinacea extracts can be \\
\hline 94 & $\begin{array}{l}\text { stimula- } \\
\text { tion }\end{array}$ & (incl. & treatment & $\mathrm{CCT}$ & $y / n$ & $\begin{array}{l}\text { studies show immunostimulat- } \\
\text { ing }\end{array}$ & efficacious immunostimulators, but \\
\hline$[42,43]$ & & $\begin{array}{l}\text { combina- } \\
\text { tions) }\end{array}$ & & & & effects & $\begin{array}{l}\text { evidence insufficient for recommen- } \\
\text { dations }\end{array}$ \\
\hline \multicolumn{8}{|c|}{ Cranberries (Vaccinium macrocarpon) } \\
\hline Jepson & urinary & cranberries & placebo & $4 \mathrm{RCT}$ & $y / y / y /$ & $\begin{array}{l}\text { In } 3 \text { of } 4 \text { trials cranberries effec- } \\
\text { tive }\end{array}$ & Insufficient evidence, further research \\
\hline $98[44]$ & $\begin{array}{l}\text { tract inf. } \\
\text { (prevent) }\end{array}$ & & & & $y / n$ & $\begin{array}{l}\text { for at least one of the outcomes } \\
\text { of interest }\end{array}$ & needed \\
\hline Jepson & urinary & cranberries & & $\begin{array}{l}\mathrm{O} \\
\mathrm{RCT}\end{array}$ & $y / y /-1$ & No trials meeting the inclusion & No evidence available \\
\hline $98[45]$ & $\begin{array}{l}\text { tract inf. } \\
\text { (treatm.) }\end{array}$ & & & & $-/-$ & criteria & \\
\hline
\end{tabular}

Mistletoe (Viscum album)

Kleijnen cancer mistletoe placebo, no II $\quad$ y/y/y/ Most studies low quality. Most Insufficient evidence to recommend 
Table 3: Systematic reviews of clinical trials of herbal medicines (at least 2 reviews per herb) (Continued)

\begin{tabular}{|c|c|c|c|c|c|c|c|}
\hline $94[46]$ & & & treatment & $\begin{array}{l}\mathrm{RCT} / \\
\mathrm{CCT}\end{array}$ & $\mathrm{n} / \mathrm{n}$ & $\begin{array}{l}\text { studies show longer survival } \\
\text { with mistletoe but not the best } \\
\text { trial }\end{array}$ & mistletoe outside of clinical trials \\
\hline Kiene 89 & cancer & mistletoe & $\begin{array}{l}\text { no treat- } \\
\text { ment, }\end{array}$ & $\begin{array}{l}2 \mathrm{RCT} \\
33\end{array}$ & $\mathrm{y} / \mathrm{n} / \mathrm{n} /$ & Most studies low quality. 9 of 12 & Available evidence supports positive \\
\hline$[47,48]$ & & & none & $\begin{array}{l}\text { CCT, } \\
\text { II } \\
\text { other } \\
\text { studies }\end{array}$ & $y / n$ & $\begin{array}{l}\text { interpretable studies suggest } \\
\text { positive effects on survival }\end{array}$ & effects of mistletoe \\
\hline \multicolumn{8}{|c|}{ Peppermint (Mentha piperita) } \\
\hline Jailwala & irritable & $\begin{array}{l}\text { I. pepper- } \\
\text { mint }\end{array}$ & placebo & $\begin{array}{l}\text { I. } 3 \\
\text { RCT }\end{array}$ & $\mathrm{p} / \mathrm{y} / \mathrm{y} /$ & $\begin{array}{l}\text { Chinese herbal therapy trial rat- } \\
\text { ed }\end{array}$ & In both cases efficacy not clearly \\
\hline $2000^{*}$ & bowel & oil & & $\begin{array}{l}\text { 2. I } \\
\text { RCT }\end{array}$ & $\mathrm{n} / \mathrm{n}$ & as positive, one of three & established \\
\hline [49] & syndr. & $\begin{array}{l}\text { 2. Chinese } \\
\text { herbal } \\
\text { therapy }\end{array}$ & & & & $\begin{array}{l}\text { peppermint oil trials rated as } \\
\text { positive }\end{array}$ & \\
\hline $\begin{array}{l}\text { Pittler } 98 \\
{[50]}\end{array}$ & $\begin{array}{l}\text { irritable } \\
\text { bowel } \\
\text { syndr. }\end{array}$ & $\begin{array}{l}\text { peppermint } \\
\text { oil }\end{array}$ & $\begin{array}{l}\text { placebo, } \\
\text { other } \\
\text { treatment }\end{array}$ & $8 \mathrm{RCT}$ & $\begin{array}{l}y / y / y / \\
y / y\end{array}$ & $\begin{array}{l}\text { Global improvement rates } \\
\text { significantly higher compared to } \\
\text { placebo. Quality of trials doubt- } \\
\text { ful }\end{array}$ & $\begin{array}{l}\text { The role of peppermint oil for IBS } \\
\text { has not been established beyond } \\
\text { reasonable doubt }\end{array}$ \\
\hline \multicolumn{8}{|c|}{ Saw palmetto (Serenoa repens) } \\
\hline Boyle & ben. & Permixon $^{\circledR}$ & placebo, & $\begin{array}{l}\text { II } \\
\text { RCTs, }\end{array}$ & $? / \mathrm{n} / \mathrm{n} /$ & $\begin{array}{l}\text { peak urine flow } 2.20(95 \% \mathrm{Cl} \\
\mathrm{I} .20-\end{array}$ & Despite some limitations strong \\
\hline $\begin{array}{l}2000 \\
{[51]}\end{array}$ & $\begin{array}{l}\text { prostate } \\
\text { hyperpla- } \\
\text { sia }\end{array}$ & $\begin{array}{l}\text { (saw } \\
\text { palmetto) }\end{array}$ & $\begin{array}{l}\text { other } \\
\text { treatment }\end{array}$ & 2 UCS & $y / y$ & $\begin{array}{l}\text { 3.20) } \mathrm{ml} / \mathrm{s} \text { increase over place- } \\
\text { bo; } \\
\text { significant decrease nocturia }\end{array}$ & $\begin{array}{l}\text { evidence that the extract tested has } \\
\text { beneficial effects }\end{array}$ \\
\hline $\begin{array}{l}\text { Wilt } \\
2000\end{array}$ & ben. & $\begin{array}{l}\text { saw palmet- } \\
\text { to }\end{array}$ & placebo, & $\begin{array}{l}14 \\
\text { RCT }\end{array}$ & $y / y / y l$ & $\begin{array}{l}\text { Saw palmetto superior to place- } \\
\text { bo }\end{array}$ & Evidence suggests that saw \\
\hline$\& 98$ & prostate & & other & (plac), & $y / y$ & $\begin{array}{l}\text { for nocturia, self rating, peak } \\
\text { urine }\end{array}$ & palmetto improves urological \\
\hline$[52,53]$ & $\begin{array}{l}\text { hyperpla- } \\
\text { sia }\end{array}$ & & treatment & $\begin{array}{l}5 \text { RCT } \\
\text { (oth- } \\
\text { er) }\end{array}$ & & $\begin{array}{l}\text { flow; similar effects as finas- } \\
\text { teride }\end{array}$ & $\begin{array}{l}\text { symptoms and flow measures. } \\
\text { Further studies needed }\end{array}$ \\
\hline
\end{tabular}


Table 4: Systematic reviews of clinical trials of herbal medicines

\begin{tabular}{|c|c|c|c|c|c|c|c|}
\hline $\begin{array}{l}\text { Author } \\
\text { Year }\end{array}$ & Indication & Intervention & Comparisons & Studies & $\begin{array}{c}\text { Features } \\
\text { I/2/3/ } \\
4 / 5\end{array}$ & Results & Author's Conclusion \\
\hline $\begin{array}{l}\text { Vogler } \\
99\end{array}$ & various & aloe & $\begin{array}{l}\text { placebo, oth- } \\
\text { er }\end{array}$ & $\begin{array}{l}6 \\
\mathrm{RCT}, 4\end{array}$ & $y / y / y /$ & Positive results for genital & Promising results, but overall \\
\hline$[54]$ & & & $\begin{array}{l}\text { \& no treat- } \\
\text { ment }\end{array}$ & $\mathrm{CCT}$ & $y / n$ & $\begin{array}{l}\text { herpes, psoriasis, hyper-lipi- } \\
\text { demia, diabetes; contradictory } \\
\text { for wound healing }\end{array}$ & evidence insufficient \\
\hline $\begin{array}{l}\text { Pittler } \\
98\end{array}$ & $\begin{array}{l}\text { choles- } \\
\text { terol low- } \\
\text { ering }\end{array}$ & $\begin{array}{l}\text { artichoke } \\
\text { leave } \\
\text { extract }\end{array}$ & placebo & I RCT & $y / y / y /$ & Effects over placebo only in the & More trials needed \\
\hline [55] & & & & & $\mathrm{n} / \mathrm{n}$ & $\begin{array}{l}\text { subgroup of participants with } \\
\text { serum cholesterol }>210 \mathrm{mg} / \mathrm{dl}\end{array}$ & \\
\hline $\begin{array}{l}\text { Morse } \\
89\end{array}$ & atopic & evening & placebo & 9 & $? / \mathrm{n} / \mathrm{n} /$ & Epogam significantly better & No conclusion drawn \\
\hline$[56]$ & eczema & $\begin{array}{l}\text { primrose oil } \\
\text { (Epogam) }\end{array}$ & & $\begin{array}{l}\mathrm{RCT} / \\
\mathrm{CCT}\end{array}$ & $y / y$ & than placebo for most outcomes & \\
\hline $\begin{array}{l}\text { Vogler } \\
98\end{array}$ & migraine & feverfew & placebo & $5 \mathrm{RCT}$ & $y / y / y /$ & Majority of trials favor feverfew & Effectiveness has not been \\
\hline$[57]$ & & & & & $y / n$ & over placebo & $\begin{array}{l}\text { established beyond reasonable } \\
\text { doubt }\end{array}$ \\
\hline $\begin{array}{l}\text { Ernst } \\
2000\end{array}$ & $\begin{array}{l}\text { nausea } \\
\text { and }\end{array}$ & ginger root & placebo, & $6 \mathrm{RCT}$ & $y / y / y /$ & 2 of 3 trials on postoperative & Evidence promising but insufficient \\
\hline$[58]$ & vomiting & & $\begin{array}{l}\text { metoclopra- } \\
\text { mide }\end{array}$ & & $y / p$ & $\begin{array}{l}\text { nausea positive (best negative), } \\
\text { trials on seasickness, morning } \\
\text { sickness and chemotherapy-in- } \\
\text { duced nausea positive }\end{array}$ & to draw firm conclusions \\
\hline $\begin{array}{l}\text { Vogler } \\
99\end{array}$ & various & $\begin{array}{l}\text { ginseng root } \\
\text { extract }\end{array}$ & $\begin{array}{l}\text { placebo, oth- } \\
\text { er }\end{array}$ & $16 \mathrm{RCT}$ & $\mathrm{y} / \mathrm{p} / \mathrm{y} /$ & Contradictory results re. & The efficacy of ginseng root extract \\
\hline$[59]$ & & & $\begin{array}{l}\text { treatment }(\mathrm{I} \\
\text { trial) }\end{array}$ & & $y / n$ & $\begin{array}{l}\text { physical performance ( } 7 \text { trials), } \\
\text { psychological function }(5) \text {, } \\
\text { immunomodulation }(2) \text {, } \\
\text { positive results in diabetes and } \\
\text { herpes simplex (I trial } \\
\text { respectively) }\end{array}$ & $\begin{array}{l}\text { is not established beyond } \\
\text { reasonable doubt for any of these } \\
\text { indications }\end{array}$ \\
\hline $\begin{array}{l}\text { Pittler } \\
98\end{array}$ & venous & horse & $\begin{array}{l}\text { placebo, oth- } \\
\text { er }\end{array}$ & $13 \mathrm{RCT}$ & $y / y / y /$ & Significant effects over placebo & horse chestnut seeds seem to be \\
\hline$[60]$ & $\begin{array}{l}\text { insuffi- } \\
\text { cieny }\end{array}$ & chestnut & treatment & & $y / n$ & and similar effects compared to & effective; further tials needed \\
\hline & & seeds & & & & other treatments & $\begin{array}{l}\text { (confirmation, long-term results, } \\
\text { combination) }\end{array}$ \\
\hline $\begin{array}{l}\text { Pittler } \\
2000\end{array}$ & anxiety & kava & placebo & $7 \mathrm{RCT}$ & $y / y / y /$ & All trials suggest superiority & Available data suggest that kava is \\
\hline$[6 \mid]$ & & & & & $\mathrm{p} / \mathrm{p}$ & $\begin{array}{l}\text { over placebo; } 3 \text { trials with data } \\
\text { for meta-analysis show sign. } \\
\text { superiority }\end{array}$ & $\begin{array}{l}\text { a treatment option for anxiety. } \\
\text { Further studies needed }\end{array}$ \\
\hline $\begin{array}{l}\text { Law- } \\
\text { rence }\end{array}$ & liver & milk thistle & $\begin{array}{l}\text { placebo, oth- } \\
\text { er }\end{array}$ & $\begin{array}{l}33 \\
\mathrm{RCT}\end{array}$ & $y / y / y /$ & Variety of conditions studied, & Efficacy is not established. \\
\hline $\begin{array}{l}2000 \\
{[62]}\end{array}$ & diseases & & $\begin{array}{l}\text { \& no treat- } \\
\text { ment }\end{array}$ & $\mathrm{I} C \mathrm{CT}$ & $y / y$ & studies often poor quality. & Possible benefit shown most \\
\hline & & & & & & Mixed and inconsistent findings & frequently for aminotransferases. \\
\hline Ernst 99 & $\begin{array}{l}\text { muscu- } \\
\text { loskel. }\end{array}$ & $\begin{array}{l}\text { Phytodo- } \\
\text { lor }^{\circledR}\end{array}$ & $\begin{array}{l}\text { placebo, oth- } \\
\text { er }\end{array}$ & I0 RCT & $y / p / y /$ & Placebo-controlled trials show & The data suggest that the \\
\hline [63] & pain & $\begin{array}{l}\text { populus, } \\
\text { fraxinus, } \\
\text { solidago }\end{array}$ & treatments & & $y / n$ & $\begin{array}{l}\text { superiority over placebo and } \\
\text { similar effects as NSAIDs }\end{array}$ & $\begin{array}{l}\text { combination is effective in the } \\
\text { symptomatic treatment of } \\
\text { muskuloskeletal pain }\end{array}$ \\
\hline Mac- & ben. pros- & rye grass & placebo, oth- & $4 \mathrm{RCT}$ & $y / y / y /$ & Signif. improvement over & Available evidence suggests that \\
\hline
\end{tabular}


Table 4: Systematic reviews of clinical trials of herbal medicines (Continued)

\begin{tabular}{|c|c|c|c|c|c|c|c|}
\hline $2000 \&$ & $\begin{array}{l}\text { hyperpla- } \\
\text { sia }\end{array}$ & pollen & therapy & & $y / y$ & placebo in subjective, but not & Cernilton $^{\circledR}$ is well tolerated and \\
\hline Wilt & & extract & & & & objective symptoms; no & modestly improves subjective \\
\hline \multicolumn{8}{|r|}{ 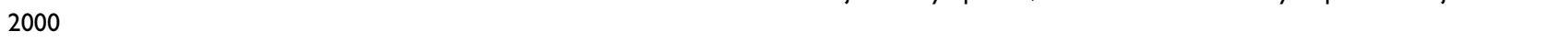 } \\
\hline$[64,65]$ & & & & & & $\begin{array}{l}\text { differences compared to } \\
\text { tadenan and paraprost }\end{array}$ & symptoms. Further studies needed \\
\hline $\begin{array}{l}\text { Ernst } \\
2000\end{array}$ & $\begin{array}{l}\text { dermato- } \\
\text { logic }\end{array}$ & tea trea oil & $\begin{array}{l}\text { placebo, } \\
\text { other }\end{array}$ & $4 \mathrm{RCT}$ & $y / y / y /$ & 2 trials vs. placebo positive, 3 & Data promising but insufficient \\
\hline \multirow[t]{2}{*}[66]{} & $\begin{array}{l}\text { condi- } \\
\text { tions }\end{array}$ & & treatment & & $y / n$ & trials vs. other treatments & \\
\hline & & & & & & similar effects & \\
\hline $\begin{array}{l}\text { Stevin- } \\
\text { son }\end{array}$ & insomnia & $\begin{array}{l}\text { valerian } \\
\text { root }\end{array}$ & placebo & $9 \mathrm{RCT}$ & $y / y / y l$ & Highly heterogeneous studies & Available evidence is promising but \\
\hline \multicolumn{7}{|l|}{ [67] } & not fully conclusive. Further, \\
\hline & & & & & & and inconsistent findings & rigorous trials needed \\
\hline $\begin{array}{l}\text { Renfrew } \\
84[68]\end{array}$ & $\begin{array}{l}\text { breast } \\
\text { engorge- } \\
\text { ment }\end{array}$ & $\begin{array}{l}\text { cabbage } \\
\text { leaves }\end{array}$ & usual care & I RCT & $\begin{array}{l}y / y / n / \\
y / n\end{array}$ & $\begin{array}{l}\text { fewer women stopping breast } \\
\text { feeding among those receiving } \\
\text { cabbage leaves }\end{array}$ & Further research desirable \\
\hline $\begin{array}{l}\text { Arm- } \\
\text { strong }\end{array}$ & atopic & Chinese & placebo & $2 \mathrm{RCT}$ & $y / y / n /$ & 2 positive studies by the same & Evidence encouraging but \\
\hline $99[69]$ & eczema & $\begin{array}{l}\text { herbal } \\
\text { therapy }\end{array}$ & & & $y / n$ & treat analysis & $\begin{array}{l}\text { insufficient given the potential of } \\
\text { relevant side effects }\end{array}$ \\
\hline Ernst 97 & hypoglyc. & all plants & no treatment, & $\begin{array}{l}7 \mathrm{RCT}, \\
4\end{array}$ & $\mathrm{y} / \mathrm{p} / \mathrm{n} /$ & Most studies low quality. Most & Use of hypoglcemic plant remedies \\
\hline \multirow[t]{2}{*}{ [70] } & activity & & $\begin{array}{l}\text { placebo, } \\
\text { none }\end{array}$ & $\begin{array}{l}\text { CCT, } \\
10\end{array}$ & $y / n$ & papers report positive effects & not supported by rigorous \\
\hline & & & & UCS & & on a variety of plants & research. Further studies required \\
\hline $\begin{array}{l}\text { Ernst } \\
2000\end{array}$ & $\begin{array}{l}\text { analgetic } \\
\text { or }\end{array}$ & various & placebo & $18 \mathrm{RCT}$ & $y / y / y l$ & Trials on evening primrose oil, & The results suggest that several \\
\hline [7I] & $\begin{array}{l}\text { inflamm. } \\
\text { treatment }\end{array}$ & & & & $y / n$ & $\begin{array}{l}\text { blackcurrant seed oil, borage } \\
\text { oil, harpagophytum, willow } \\
\text { bark, feverfew, and } 3 \\
\text { combinations; almost all trials } \\
\text { positive }\end{array}$ & $\begin{array}{l}\text { herbal remedies have potential in } \\
\text { alleviating the pain of rheumatic } \\
\text { diseases. More research urgently } \\
\text { needed }\end{array}$ \\
\hline
\end{tabular}

legend see table I

\section{Discussion}

Our overview shows that a considerable number of systematic reviews on herbal medicines is available. In the majority of cases the reviewers considered the available evidence as promising but only very rarely as convincing and sufficient as a firm basis for clinical decisions. The methodological quality of the primary studies has been criticized by many reviewers.

Our summary of the existing studies must be interpreted with caution. What we performed is a systematic review of systematic reviews which inherently bears a large risk of oversimplification. Readers who want to reliably assess the evidence for a given herb for a defined condition should read the respective reviews. Our collection which to the best of our knowledge is complete up to summer 2000 - is aimed at facilitating the access and giving an idea of the amount of the available evidence.
Based on the increase of herbal medicine reviews in recent years we expect that at least ten new publications will become available in the year 2001 .

Most of the currently available systematic reviews address herbal preparations which are marketed and widely used in industrialized countries. However, the widespread traditional use of herbs in the Third World is rarely ever investigated and has not been subjected to systematic reviews. The many herbs used in folk medicine or other traditional uses of herbs (for example, hypericum is used for a variety of ailments other than depression including enuresis, diarrhoea, gastritis, bronchitis, asthma, sleeping disorders etc.) seem to be rarely investigated. Furthermore, practitioners of herbal medicine often combine different herbs and use unconventional diagnostic approaches to adapt prescriptions to single patients. It seems likely that these traditional 
forms of herbal medicine will remain underresearched relative to single herbal preparations due to the lack of financial incentive for sponsors and due to methodological problems.

Herbal medicines products are not, in general, subject to patent protection. This reduces the motivation for drug companies to invest in trials. Many of the existing herbal medicine manufacturers are comparably small companies, often with limited research resources and expertise. Maybe partly for these reasons, the quality of many older herbal medicine trials is low. Furthermore, negative trials which could threaten the company's survival might not become published.

A fundamental problem in all clinical research of herbal medicines is whether different products, extracts, or even different lots of the same extract are comparable and equivalent. This is a major issue in the expert research community and a major obstacle to a reliable assessment for the non-expert. For example, Echinacea products can contain other plant extracts, use different plant species (E. purpurea, pallida or angustifolia), different parts (herb, root, both), and might have been produced in quite different manners (hydro- or lipophilic extraction). Pooling studies that use different herbal products in a quantitative meta- analysis can be misleading. Health care professionals and patients considering to prescribe or take a particluar herbal product should check carefully whether the respective product or extract has been tested in the trials included in a review. On the health food store shelf the high quality, standardized products used in the trials might not be available. Only a herbal medicine expert can judge with some certainty whether the results can be extrapolated to the product of interest.

On the level of health care policies the available systematic reviews more often provide insight into the deficiencies of the evidence than guidance for decision making. Trials on hard endpoints are very rarely available and observation periods have generally been short. The clinical relevance of the observed effects is not always clear.

Herbal medicines are generally considered as comparably safe. While this is probably correct case reports show that severe side effects and relevant interactions with other drugs can occur. For example, hypericum extracts cause considerably fewer side effects than tricyclic antidepressants [92] but can decrease the concentration of a variety of other drugs by enzyme induction [93]. Several reviews summarizing side effects and interactions have been published [94-98].
In conclusion, the systematic reviews collected for this analysis are a good tool to get an overview of the available evidence from clinical trials in the area of herbal medicine. However, applying the findings to patients care is problematic for those who are not experts in herbal medicine. In this case it might be better to directly search the literature for clinical trials of the respective product.

\section{Competing interest}

$\mathrm{KL}, \mathrm{DM}, \mathrm{GtR}$, and AV have been involved in some of the reviews analyzed. These were extracted and assessed by other members of the team.

\section{Acknowledgements}

KL's work was partly funded by the NIAMS grant 5 U24-AR-43346-02 and by the Carl and Veronica Carstens Foundation, Essen, Germany. We would like to thank Brian Berman for his support, his help to get funding and his patience in awaiting the completion of our work.

\section{References}

I. Vickers A, Zollman CE: ABC of complementary medicine: herbal medicine $B M$ J 1999, 3 19:1050-1053

2. Schwabe U: Arzneimittel der besonderen Therapierichtungen (Naturheilmittel) In Arzneiverordnungs-Report 1998. Edited by Schwabe U, Paffrath D. Berlin: Springer, 199962 I-656

3. Brevoort P: The booming US botanical market. A new overview HerbalGram 1998, 44:33-5 I

4. Barnes J: Phytotherapy: consumer and pharmacist perspectives In: Herbal medicine - a concise overview for professionals. Edited by Ernst E. Oxford: Butterworth Heinemann, 200019-33

5. Bodeker GC: Editorial J Altem Complement Med I996, 3:323-326

6. Linde K, Vickers A, Hondras M, et al: Systematic reviews of complementary therapies - an annotated bibliography. Part I: acupuncture BMC Complementary and Alternative Medicine. 200I, I:3:

7. Pittler $\mathrm{MH}$, Ernst E: Ginkgo biloba extract for the treatment of intermittent claudication: a meta- analysis of randomized trials Am J Med 2000, I08:276-28I

8. Moher D, Pham B, Ausejo M, Saenz A, Hood S, Barber GG: Pharmacological management of intermittent claudication: a metaanalysis of randomised trials Drugs 2000, 59:1057-1070

9. Ernst E: Ginkgo biloba in der Behandlung der Claudicatio intermittens Fortschr Med 1996, I I 4:85-87

10. Schneider B: Ginkgo-biloba-Extrakt bei peripheren arteriellen Verschlusskrankheiten. Meta Analyse von kontrollierten klinischen Studien Arzneim-Forsch/Drug Res 1992, 42(I):428-436

II. Letzel H, Schoop W: Gingko-biloba-Extrakt EGb 76 I und Pentoxifyllin bei Claudicatio intermittens. Sekundäranalyse zur klinischen Wirksamkeit VASA 1992, 2 I:403-4 I0

12. Kleijnen J, Knipschild P: Gingko biloba for intermittent claudication and cerebral insufficiency In: Kleijnen J. Food supplements and their efficacy. Maastricht: Rijksuniversiteit Limburg, 199183-94

13. Weiss G, Kallischnigg G: Gingko-biloba-Extrakt (EGb 76I) Meta-Analyse von Studien zum Nachweis der therapeutischen Wirksamkeit bei Hirnleistungsstorungen bzw. peripherer arterieller Verschlusskrankheit Muench med Wschr 1991, I 0:138-142

14. Ernst E, Pittler MH: Ginkgo biloba for dementia: a systematic review of double-blind, placebo- controlled trials Clin Drug Invest 1999, 17:301-308

15. Oken BS, Storzbach DM, Kaye JA: The efficacy of ginkgo biloba on cognitive function in Alzheimer disease Arch Neurol 1998, 55:1409-1415

16. Hopfenmuller W: Nachweis der therapeutischen Wirksamkeit eines Ginkgo biloba-Spezialextraktes - Meta-Analyse von I I klinischen Studien mit Patienten mit Hirnleistungsstörungen im Alter Arzneim-Forsch /Drug Res 1994, 44(II): I 005- I 013

17. Kleijnen J, Knipschild P: Gingko biloba for cerebral insufficiency $\mathrm{Br}$ 」 din Pharmacol 1992, 34:352-358

18. Ernst E, Stevinson C: Ginkgo biloba for tinnitus: a review Clin Otolaryngol 1999, 24:164-167 
19. Evans JR: Ginkgo biloba extract for age-related macular degeneration (Cochrane Review) In: The Cochrane Library, Issue I, 2000. Oxford: Update Software.

20. Gaster B: St John's wort for depression. A systematic review Arch Intern Med 2000, I 60:152-156

21. Williams JW jr, Mulrow CD, Chiquette E, Hitchcock Noel P, Aguilar $C$, Cornell ]: A systematic review of newer pharmacotherapies for depression in adults: Evidence report summary Ann Int Med 2000, I 32:743-756

22. Mulrow CD, Williams JW jr, Trivedi M, et al: Treatment of depression - newer pharmacotherapies Psychopharmacol Bull 1998, 34:409-795

23. Kim HL, Streltzer J, Goebert D: St. John's wort for depression: A meta-analysis of well-defined clinical trials J Nerv Ment Dis 1999, I 87:532-539

24. Stevinson C, Ernst E: Hypericum for depression. An update of the clinical evidence Neuropsychopharmacol 1999, 9:501-505

25. Linde K, Mulrow CD: St John's wort for depression (Cochrane Review) In: The Cochrane Library, Issue 4, 1998. Oxford: Update Software.

26. Linde K, Ramirez G, Mulrow CD, Pauls A, Weidenhammer W, Melchart D: St John's wort for depression - an overview and meta-analysis of randomised clinical trials $B M$ J I 996, 3 I 3:253258

27. Volz HP: Controlled clinical trials of hypericum extract in depressed patients - an overview Pharmacopsychiat 1997, 30 (suppl):72-75

28. Ernst E: St. John's Wort, an anti-depressant? A systematic, criteria-based review Phytomedicine 1995, 2:67-7|

29. Volz HP, Laux P: Potential treatment for subthreshold and mild depression: A comparison of St. John's wort extracts and fluoxetine Comprehensive Psychiatry 2000, 4 I (suppl I):I33137

30. Friede M, Wüstenberg P: Johanniskraut zur Therapie von Angstsyndromen bei depressiven Verstimmungen Ztschr Phytother 1998, 19:309-317

31. Lawrence V, Mulrow C, Ackerman R, et al: Garlic: Effects on cardiovascular risks and disease, protective effects against cancer, and clinical adverse effects Evidence Report/Technology Assessment: Number 20. 2000 [http://www.ahcpr.gov/clinic/garlicsum.htm]

32. Stevinson C, Pittler MH, Ernst E: Garlic for treating hypercholesterinemia - a meta-analysis of randomized clinical trials Ann Intern Med 2000, 133:420-429

33. Silagy C, Neil A: Garlic as a lipid lowering agent - a meta-analysis J Roy Coll Phys 1994, 28:39-45

34. Neil HAW, Silagy CA, Lancaster, et al: Garlic powder in the treatment of moderate hyperlipidaemia: a controlled trial and meta-analysis J Roy Coll Pract London 1996, 30:329-334

35. Warshafsky S, Kamer RS, Sivak SL: Effect of garlic on total serum cholesterol Ann Int Med 1993, I 19:599-605

36. Silagy CA, Neil HA: A meta-analysis of the effect of garlic on blood pressure J Hypertension 1994, I 2:463-468

37. Kleijnen J: Controlled clinical trials in humans on the effects of garlic supplements In: Kleijnen J. Food supplements and their efficacy. Maastricht: Rijksuniversiteit Limburg, 199173-82

38. Kleijnen J, Knipschild P, ter Riet G: Garlic, onions and cardiovascular risk factors. A review of the evidence from human experiments with emphasis on commercially available preparations $\mathrm{Br}$ J Clin Pharmacol 1989, 28:535-544

39. Jepson RG, Kleijnen J, Leng GC: Garlic for lower limb atherosclerosis (Cochrane Review) In: The Cochrane Library, Issue 4, 1998. Oxford: Update Software.

40. Barrett B, Vohmann M, Calabrese C: Echinacea for upper respiratory tract infection J Fam Pract 1999, 48:628-635

4I. Melchart D, Linde K, Fischer P, Kaesmayr J: Echinacea for prevention and treatment of the common cold (Cochrane Review) In: The Cochrane Library, Issue I, 1999. Oxford: Update Software.

42. Melchart D, Linde K, Worku F, Bauer R, Wagner H: Immunmodulation mit Echinacea-haltigen Arzneimittein - eine kriteriengestützte Analyse der kontrollierten klinischen Studien Forsch Komplementärmed 1994, 1:26-36

43. Melchart D, Linde K, Worku F, Bauer R, Wagner H: Immunomodulation with Echinacea - a systematic review of controlled clinical trials Phytomedicine 1994, I:245-254
44. Jepson RG, Mihaljevic L, Craig J: Cranberries for the prevention of urinary tract infections (Cochrane Review) In The Cochrane Library, Issue 4, 1998. Oxford: Update Software.

45. Jepson RG, Mihaljevic L, Craig J: Cranberries for the treatment of urinary tract infections (Cochrane Review) In The Cochrane Library, Issue 4, 1998. Oxford: Update Software.

46. Kleijnen J, Knipschild P: Mistletoe treatment for cancer. Review of controlled trials in humans Phytomedicine 1994, I:255-260

47. Kiene H: Klinische Studien zur Misteltherapie karzinomatöser Erkrankungen Therapeutikon 1989, 6:347-353

48. Kiene H: Klinische Studien zur Misteltherapie der Krebserkrankung. Eine kritische Würdigung Herdecke: Dissertation, 1989

49. Jailwala J, Imperiale TF, Kroenke K: Pharmacologic treatment of the irritable bowel syndrome: a systematic review of randomized, controlled trials Ann Intern Med 2000, I33:I36-147

50. Pittler $\mathrm{MH}$, Ernst E: Peppermint oil for irritable bowel syndrome: a critical review and meta- analysis $A m$ J Gastroenterol 1998, 93:1131-1135

5I. Boyle P, Robertson C, Lowe F, Roehborn C: Meta-analysis of clinical trials of Permixon in the treatment of symptomatic benign prostatic hyperplasia Urology 2000, 55:533-539

52. Wilt TJ, Ishani A, MacDonald R, Stark G, Mulrow C, Lau J: Serenoa repens for treatment of benign prostatic hyperplasia (Cochrane Review) In: The Cochrane Library, Issue I, 2000. Oxford: Update Software.

53. Wilt T], Ishani A, Stark G, MacDonald R, Lau J, Mulrow C: Saw palmetto extracts for treatment of benign prostatic hyperplasia - a systematic review JAMA 1998, 280:1604-I609

54. Vogler BK, Ernst E: Aloe vera: A systematic review of its clinical effectiveness Br J Gen Pract 1999, 49:823-828

55. Pittler $\mathrm{MH}$, Ernst $\mathrm{E}$ : Artichoke leaf extract for serum cholesterol reduction Perfusion 1998, I I:338-340

56. Morse PF, Horrobin DF, Manku MS, et al: Meta-analysis of placebo-controlled studies of the efficacy of Epogam in the treatment of atopic eczema. Relationship between plasma essential fatty acid changes and responses $\mathrm{Br} J$ Dermatol 1989, | 2 |:75-90

57. Vogler BK, Pittler MH, Ernst E: Feverfew as a preventive treatment for migraine: a systematic review Cephalalgia 1998, I 8:704-708

58. Ernst E, Pittler MH: Efficacy of ginger for nausea and vomiting: a systematic review of randomized clinical trials Intern J Anesth 2000, 84:367-37।

59. Vogler BK, Pittler MH, Ernst E: The efficacy of ginseng. A systematic review of randomised clinical trials Eur J Clin Pharmaco 1999, 55:567-575

60. Pittler $\mathrm{MH}$, Ernst $\mathrm{E}$ : Horse-chestnut seed extract for chronic venous insufficiency Arch Dermatol 1998, I 34: I 356-1 360

6I. Pittler MH, Ernst E: Efficacy of kava extract for treating anxiety: systematic review and meta- analysis J Clin Psychopharmacol 2000, 20:84-89

62. Lawrence V, Mulrow C, Jacobs B, et al: Report on milk thistle: Effects on liver disease and cirrhosis and clinical adverse effects Evidence Report/Technology Assessment: Number 21. [http://www.ahcpr.gov/clinic/milktsum.htm]

63. Ernst E: The efficacy of Phytodolor for the treatment of musculoskeletal pain - a systematic review of randomized clinical trials Natural Medicine Journal 1999, (Summer): |4-7

64. MacDonald R, Ishani A, Rutks I, Wilt TJ: A systematic review of Cernilton for the treatment of benign prostatic hyperplasia Br J Urol Int 2000, 85:836-84I

65. Wilt TJ, MacDonald R, Ishani A, Rutks I, Stark G: Cernilton for benign prostatic hyperplasia (Cochrane Review) In: The Cochrane Library, Issue 2, 2000. Oxford: Update Software.

66. Ernst $E$, Huntley A: Tea tree oil: a systematic review of randomised clinical trials Forsch Komplementärmed 2000, 7:I7-20

67. Stevinson C, Ernst E: Valerian for insomnia: a systematic review of randomized clinical trials Medicine 2000, I:9|-99

68. Renfrew MJ, Lang S: Do cabbage leaves prevent breast engorgement? (Cochrane Review) In: The Cochrane Library, Issue 4, 1998. Oxford: Update Software.

69. Armstrong NC, Ernst E: The treatment of eczema with Chinese herbs: a systematic review of randomized controlled trials $\mathrm{Br}$ J Clin Pharmacol 1999, 48:262-264

70. Ernst E: Plants with hypoglycaemic activity in humans Phytomedicine 1997, 4:73-78 
7I. Ernst E, Chrubasik S: Phyto-anti-inflammatories: a systematic review of randomized, placebo- controlled, double-blind trials Rheum Dis Clin North America 2000, 26:13-27

72. Budeiri D, Li Wan Po A, Dornan JC: Is Evening Primrose Oil of value in the treatment of premenstrual syndrome? Controlled Clin Trials 1996, 17:60-68

73. Diehm C: The role of oedema protective drugs in the treatment of venous insufficiency: a review of evidence based on placebo-controlled clinical trials with regard to efficacy and tolerance Phlebology 1996, I I:23-29

74. Evans MF, Morgenstern K: St. John's wort: an herbal remedy for depression? Canadian Family Physician 1997, 43:1735-1736

75. Giles JT, Palat CR, Chien SH, Chang ZG, Kennedy DT: Evaluation of echinacea for treatment of the common cold Pharmacotherapy 2000, 20:690-697

76. Josey ES, Tackett RL: St. John's wort: a new alternative for depression? Intern J Clin Pharmacol Ther 1999, 37: I I I-II 9

77. Kleijnen J, ter Riet G, Knipschild P: Teunisbloemolie. Een overzichtvan gecontroleerd onderzoek Pharmaceutisch Weekblad 1989, I 24:418-423

78. Knipschild P: Ginseng: pep of nep? Pharmaceutisch Weekblad 1988, | 23:4-II

79. McPartland JM, Pruitt PL: Medical marijuana and its use by the immunocompromised Altern Ther Health Med 1997, 3:39-45

80. Weihmayr T, Ernst E: Die therapeutische Wirksamkeit von Crataegus Fortschr Med 1996, II 4:27-29

81. Wettstein A: Cholinesterase inhibitors and ginkgo extracts are they comparable in the treatment of dementia? Comparison of published placebo-controlled efficacy studies of at least six months duration Phytomed 2000, 6:393-40I

82. Wong $\mathrm{AHC}$, Smith $\mathrm{M}$, Boon HS: Herbal remedies in psychiatric practice Arch Gen Psychiatry 1998, 55:1033-1044

83. Ernst E, Pittler MH: Yohimbine for erectile dysfunction: a systematic review and meta-analysis of randomized clinical trials J Urol 1998, 159:433-436

84. Mclntosh HM, Olliaro P: Artemisin derivatives for treating uncomplicated malaria (Cochrane Review) In: The Cochrane Library, Issue 2, 2000. Oxford: Update Software.

85. Mclntosh HM, Olliaro P: Artemisin derivatives for treating severe malaria (Cochrane Review) In: The Cochrane Library, Issue 2, 2000. Oxford: Update Software.

86. Pittler $\mathrm{MH}$, Ernst E: Artemether for severe malaria: a metaanalysis of randomized clinical trials Clin Infect Dis 1999, 28:597601

87. Wilt TJ, Ishani A, MacDonald R, Stark G, Mulrow C, Lau J: Beta-sitosterols for benign prostatic hyperplasia (Cochrane Review) In: The Cochrane Library, Issue I, 2000. Oxford: Update Software.

88. Dumont L, Mardirosoff C. Tramér M: Efficacy and harm of pharmacological prevention of acute mountain sickness: a quantiative systematic review $B M / 2000,321: 267-272$

89. Ernst E: Can allium vegetables prevent cancer? Phytomed I997, 4:79-83

90. Joy CB, Mumby-Croft R, Joy LA: Polyunsaturated fatty acid (fish or evening primrose oil) for schizophrenia (Cochrane Review) In: The Cochrane Library, Issue 2, 2000. Oxford: Update Software.

91. Young GL, Jewell MD: Creams to prevent striae gravidarum (Cochrane Review) In: The Cochrane Library, Issue 4, 1998. Oxford: Update Software.

92. Ernst E, Rand JI, Barnes J, Stevinson C: Adverse effects profile of the herbal antidepressant St. John's wort (Hypericum perforatum L.) Eur I Clin Pharmacol I 998, 54:589-594

93. Ernst E: Second thoughts about safety of St John's wort Lancet 1999, 354:2014-2015

94. De Smet PAGM: Health risks of herbal remedies Drug Safety 1995, I 3:81-93

95. Miller LG: Herbal medicine. Selected clinical considerations focusing on known or potential drug- herb interactions Arch Intern Med 1998, 158:2200-22II

96. Fugh-Berman A: Herb-drug interactions Lancet 2000, 355:134138

97. Ernst E: Possible interactions between synthetic and herbal medicinal products. Part I: a systematic review of the indirect evidence Perfusion 2000, 13:4-15

98. Ernst E: Possible interactions between synthetic and herbal medicinal products. Part 2: a systematic review of the direct evidence Perfusion 2000, 13:60-70

\section{Pre-publication history}

The pre-publication history for this paper can be accessed here:

http://www.biomedcentral.com/content/backmatter/ 1472-6882-1-5-b1.pdf

Publish with BioMed Central and every scientist can read your work free of charge

"BioMedcentral will be the most significant development for disseminating the results of biomedical research in our lifetime."

Paul Nurse, Director-General, Imperial Cancer Research Fund

Publish with BMC and your research papers will be:

- available free of charge to the entire biomedical community

- peer reviewed and published immediately upon acceptance

- cited in PubMed and archived on PubMed Central

- yours - you keep the copyright 